\title{
Atividade fotossintética e produção de biomassa de clones de batata em função da disponibilidade de fósforo no solo
}

Photosynthetic activity and biomass production of potato clones as a function of soil phosphorus availability

Actividad fotosintética y producción de biomasa de clones de papa en función de la disponibilidad de fósforo en el suelo

Recebido: 18/06/2021 | Revisado: 24/06/2021 | Aceito: 27/06/2021 | Publicado: 11/07/2021

Camila Peligrinotti Tarouco

ORCID: https://orcid.org/0000-0002-9488-9773 Universidade Estadual do Norte do Paraná, Brasil E-mail:milatarouco@gmail.com

Darlene Sausen

ORCID: https://orcid.org/0000-0002-3896-0168 Agro Sausen, Brasil

E-mail: darlene_sn@yahoo.com.br Miriam da Silva Tavares

ORCID: https://orcid.org/0000-0002-4284-7790 Universidade Estadual de Goiás, Brasil

E-mail: myriann2017@gmail.com

Fernando Teixeira Nicoloso

ORCID: https://orcid.org/0000-0002-7900-5263

Universidade Federal de Santa Maria, Brasil E-mail: ftnicoloso@yahoo.com

\begin{abstract}
Resumo
Plantas de batata (Solanum tuberosum L.) possuem maior exigência por fósforo $(\mathrm{P})$ para atingirem altos rendimentos de tubérculos. Em solos deficientes em $\mathrm{P}$ a atividade fotossintética e a produtividade das culturas são consideravelmente reduzidas. Desse modo, o objetivo deste estudo foi investigar os efeitos na fotossíntese e no crescimento de clones de batata cultivados em diferentes níveis de P no solo. O experimento foi conduzido em casa de vegetação, em delineamento inteiramente casualizado, utilizando solo como substrato, onde os tratamentos foram combinados em esquema fatorial com três clones de batata (Atlantic, SMIC 148-A e SMINIA 793101-3) e quatro doses de fósforo (35, 70, 140 e $280 \mathrm{~kg}$ de P2O5 ha-1). A aplicação de doses de P alterou a produção de massa seca de tubérculos e total dos clones estudados. O clone SMIC 148-A apresentou maior taxa de assimilação líquida do CO2 e condutância estomática nas doses de 70, 140 e 280 kg P2O5 ha-1. O clone Atlantic foi o que apresentou maior produção de massa seca total na dose mais baixa de $\mathrm{P}$, bem como foi o clone que investiu os fotoassimilados de forma mais eficiente, visto que, obteve maior massa seca de tubérculos. A maior dose de fósforo foi a que proporcionou maior massa de tubérculos.
\end{abstract}

Palavras-chave: Concentração interna de $\mathrm{CO}_{2}$; Massa seca; Solanum tuberosum L.; Taxa de assimilação de $\mathrm{CO}_{2}$; Transpiração.

\begin{abstract}
Potato plants (Solanum tuberosum L.) have a higher requirement for phosphorus (P) to achieve high yields of tubers. In P-deficient soils, photosynthetic activity and crop productivity are considerably reduced. Thus, the aim of this study was to investigate the effects on photosynthesis and growth of potato clones cultivated at different levels of $\mathrm{P}$ in the soil. The experiment was conducted in a greenhouse, in a completely randomized design, using soil as substrate, where treatments were combined in a factorial scheme with three potato clones (Atlantic, SMIC 148-A and SMINIA 793101-3) and four P doses (35, 70, 140 and $280 \mathrm{~kg}$ P2O5 ha-1). The application of P doses changed THE PRODUCTION of dry matter of tubers and total of the clones studied. The SMIC 148-A clone presented a higher of net $\mathrm{CO} 2$ assimilation rate and stomatal conductance at doses of 70, 140 and $280 \mathrm{~kg}$ P2O5 ha-1. The Atlantic clone was the one that presented the highest total dry mass production in the lowest dose of $\mathrm{P}$, as well as the clone that invested the photoassimilates more efficiently, since it obtained the highest dry mass of tubers. The highest dose of phosphorus was the one that provided the largest mass of tubers.
\end{abstract}

Keywords: $\mathrm{CO}_{2}$ assimilation rate; Dry mass; Intercellular $\mathrm{CO}_{2}$ concentration; Solanum tuberosum L.; Transpiration. 


\begin{abstract}
Resumen
Las plantas de papa (Solanum tuberosum L.) tienen una alta demanda de fósforo $(\mathrm{P})$ para lograr altos rendimientos de tubérculos. En suelos deficientes en P, la actividad fotosintética y la productividad de los cultivos se reducen considerablemente. Así, el objetivo de este estudio fue evaluar los efectos sobre la fotosíntesis y el crecimiento de clones de papa sometidos a diferentes niveles de $\mathrm{P}$ en el suelo. El experimento se realizó en invernadero, en un diseño completamente al azar, utilizando tierra como sustrato, donde los tratamientos se combinaron en factorial con tres clones de papa (Atlantic, SMIC 148-A y SMINIA 793101-3) y cuatro dosis de fósforo (35, 70, 140 y $280 \mathrm{~kg}$ de P2O5 ha-1). La aplicación de dosis de $\mathrm{P}$ alteró la producción de masa seca de tubérculos y total de los clones estudiados. El clon SMIC 148-A mostró la mayor tasa de asimilación neta de CO2 y conductancia estomática a dosis de 70, 140 y $280 \mathrm{~kg}$ P2O5 ha-1. El clon Atlántico fue el que presentó mayor producción de masa seca total a la menor dosis de P, así como el clon que invirtió los fotoasimilados de manera más eficientes, ya que obtuvo la mayor masa seca de tubérculos. La dosis más altas de fósforo fue la que proporcionó la mayor masa de tubérculos.

Palabras clave: Concentración interna de $\mathrm{CO}_{2}$, Masa seca; Solanum tuberosum L.; Tasa de asimilación de $\mathrm{CO}_{2}$; Transpiración.
\end{abstract}

\title{
1. Introdução
}

A batata é uma cultura que apresenta uma extensão de pool genético, gerando uma ampla gama de opções de mercado, que variam da agricultura familiar não mecanizada em pequena escala até a produção mecanizada de média a grande escala (Haan \& Rodriguez, 2016). No entanto, como parte de uma estratégia agronômica de sucesso para obtenção de uma adequada produtividade da batata faz-se necessário o manejo adequado dos nutrientes (Koch et al., 2019).

Neste contexto, a batata é uma cultura com restrita capacidade de retirar o fósforo (P) em solos com baixa disponibilidade, por isso, normalmente são aplicadas grandes quantidades de fertilizantes fosfatados para o desenvolvimento adequado da planta e a obtenção de elevados rendimentos de tubérculos (Dechassa et al., 2003, Balemi \& Schenk, 2009).

O P é importante para o enraizamento inicial e o desenvolvimento das brotações, fornecendo energia para os processos da planta, como a absorção e o transporte de íons. Além disso, ele está envolvido em funções chaves que envolvem a conservação e transferência de energia, síntese de fotoassimilados, alocação do carbono em açúcares e amidos, translocação de nutrientes dentro da planta e transferência de características genéticas pelas gerações (Shen et al., 2011).

Como mais da metade dos solos agrícolas no mundo apresentam baixos níveis de P (Wang et al., 2010) e os processos fotossintéticos que condicionam a produtividade das culturas são dependentes da disponibilidade deste, a falta de P pode limitar a produção de biomassa. Os processos fotossintéticos são os principais, senão os únicos, processos de fornecimento de carbono para o metabolismo vegetal. Os fotoassimilados produzidos são direcionados para o crescimento, sendo a partição para os tubérculos um dos fatores determinantes da produtividade da batata (Braun et al., 2011).

A deficiência de P pode diminuir a atividade da Ribulose-1,5-bisfosfato (RuBP) ou ocasionar uma produção de ATP insuficiente (Jacob \& Lawlor, 1992; Pieters et al., 2001), devido a conversão menos eficiente da radiação interceptada (Plenet et al., 2000), comprometendo diretamente a fixação do carbono pelas plantas. Ainda, a deficiência de P pode influenciar na produtividade dependendo do tempo e da severidade dessa restrição, bem como da capacidade do genótipo em utilizar internamente o P (Santos et al., 2006).

Desse modo, o objetivo deste estudo foi investigar os efeitos na fotossíntese e no crescimento de clones de batata cultivados em diferentes níveis de P no solo.

\section{Metodologia}

O experimento foi conduzido em casa de vegetação ( $\left.29^{\circ} 42^{\prime} 56^{\prime \prime} \mathrm{S}, 53^{\circ} 43^{\prime} 13^{\prime \prime} \mathrm{O}\right)$ com temperatura parcialmente controlada, mantido em $25 \pm 3{ }^{\circ} \mathrm{C}$, durante a primavera. Foram utilizados dois clones SMIC 148-A e SMINIA 793101-3 do Programa de Genética e Melhoramento da Batata da Universidade Federal de Santa Maria - UFSM e a cultivar Atlantic, que para fins de simplificação será referida como clone. 
Para o experimento foram utilizados vasos plásticos de $5 \mathrm{~L}(20 \mathrm{~cm}$ de diâmetro por $20 \mathrm{~cm}$ de altura) que continham $4 \mathrm{~kg}$ de solo proveniente de campo nativo do tipo argissolo bruno-acinzentado alítico úmbrico (EMBRAPA, 2018). Os principais atributos desse solo são: $18 \%$ de argila; $25 \mathrm{~g} \mathrm{~kg}^{-1}$ de matéria orgânica; $\mathrm{pH}$ (água 1:1) de 4,6; 30,6\% de Al; 26,5\% de saturação de bases; 6,2 $\mathrm{cmolc} \mathrm{dm}^{-3}$ de CTC efetiva; 2,8cmolc dm ${ }^{-3}$ de Ca, 1,4cmolc dm${ }^{-3}$ de $\mathrm{Mg}, 3 \mathrm{mg} \mathrm{kg}^{-1}$ de P (Mehlich ${ }^{-1}$ ) e $76 \mathrm{mg} \mathrm{kg}^{-1} \mathrm{de}$ $\mathrm{K}\left(\right.$ Mehlich $\left.^{-1}\right)$. A correção do $\mathrm{pH}$ foi realizada através da incorporação de hidróxido de cálcio $\left(\mathrm{Ca}(\mathrm{HO})_{2}\right)$ em conjunto com óxido de magnésio $(\mathrm{MgO})$ (foi realizado teste preliminar que mostrou a eficácia desse procedimento). Três dias após a calagem realizou-se a aplicação de ureia $\left(\mathrm{CO}\left(\mathrm{NH}_{2}\right)_{2}\right)$ e cloreto de potássio $(\mathrm{KCl})$ conforme recomendado para cultivo da batata (Comissão de química e fertilidade do solo, 2004).

$\mathrm{O}$ delineamento experimental foi o inteiramente casualizado em esquema fatorial $4 \times 3$, com quatro repetições. Os

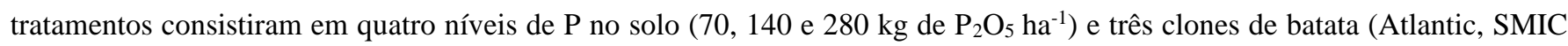
148-A e SMINIA 793101-3).

No momento do plantio os tubérculos selecionados apresentaram peso médio de 32, 26 e 28g, respectivamente para os clones Atlantic, SMIC 148-A e SMINIA 793101-3. Os níveis de P utilizados foram de 0,025;0,05; 0,11 e 0,22g de monofosfato de potássio $\left(\mathrm{KH}_{2} \mathrm{PO}_{4}\right)$, o que equivale aos níveis de 35, 70, 140 e $280 \mathrm{~kg}$ de $\mathrm{P}_{2} \mathrm{O}_{5}$ ha $^{-1}$. O monofosfato de potássio foi dissolvido em água e incorporado ao solo dez dias após o plantio dos tubérculos. A irrigação ocorreu manualmente, conforme necessário para manter o solo com 70\% da capacidade de campo através da pesagem diária dos vasos.

Aos 69 dias após o plantio, ainda em estágio de tuberização, segundo Heldwein et al. (2009), foram avaliadas as seguintes variáveis: taxa de assimilação líquida do $\mathrm{CO}_{2}\left(A-\mu \mathrm{mol} \mathrm{CO}_{2} \mathrm{~m}^{-2} \mathrm{~s}^{-1}\right)$; condutância estomática de vapores de água (Gs - mol $\mathrm{H}_{2} \mathrm{O} \mathrm{m}^{-2} \mathrm{~s}^{-1}$ ); a eficiência do uso da água (EUA - mol $\mathrm{CO}_{2} \mathrm{~mol} \mathrm{H}_{2} \mathrm{O}^{-1}$ ), obtida pela relação entre quantidade de $\mathrm{CO}_{2}$ fixado pela fotossíntese e quantidade de água transpirada; e eficiência instantânea de carboxilação da enzima ribulose-1,5bifosfato carboxilase/oxigenase (Rubisco), calculada pela relação da taxa de assimilação de $\mathrm{CO}_{2}$ e concentração interna de $\mathrm{CO}_{2}$ na folha $(A / C i)$. Essas avaliações foram realizadas na terceira folha completamente expandida da planta entre as 9 e as $11 \mathrm{~h}$, com a utilização do medidor portátil IRGA, marca LI-COR, modelo LI-6400XT, utilizando uma radiação fotossintética de $1500 \mu \mathrm{mol} \mathrm{m} \mathrm{m}^{-2} \mathrm{~s}^{-1}$ e uma concentração de $\mathrm{CO}_{2}$ de $400 \mu \mathrm{mol} \mathrm{mol}^{-1}$ (Figura 1). A luminosidade utilizada foi proveniente do próprio equipamento que possui câmara de luz acoplada e a determinação da radiação de $1500 \mu \mathrm{mol} \mathrm{m} \mathrm{m}^{-2} \mathrm{~s}^{-1}$ foi obtida através da realização da curva de luz, onde aplicou-se nove níveis de irradiância (2000, 1500, 1000, 500, 250, 120, 60, 30 e $0 \mu \mathrm{mol} \mathrm{m}^{-2}$ $\left.\mathrm{s}^{-1}\right)$ que teve como objetivo definir o ponto de compensação e saturação luminosa da cultura, sendo determinado o ponto de saturação de luz de $1500 \mu \mathrm{mol} \mathrm{m}^{-2} \mathrm{~s}^{-1}$. 
Figura 1. Avaliação da atividade fotossintética na terceira folha completamente expandida da planta de batata entre as 9 e as 11h, com a utilização do medidor portátil IRGA, marca LI-COR, modelo LI-6400XT.

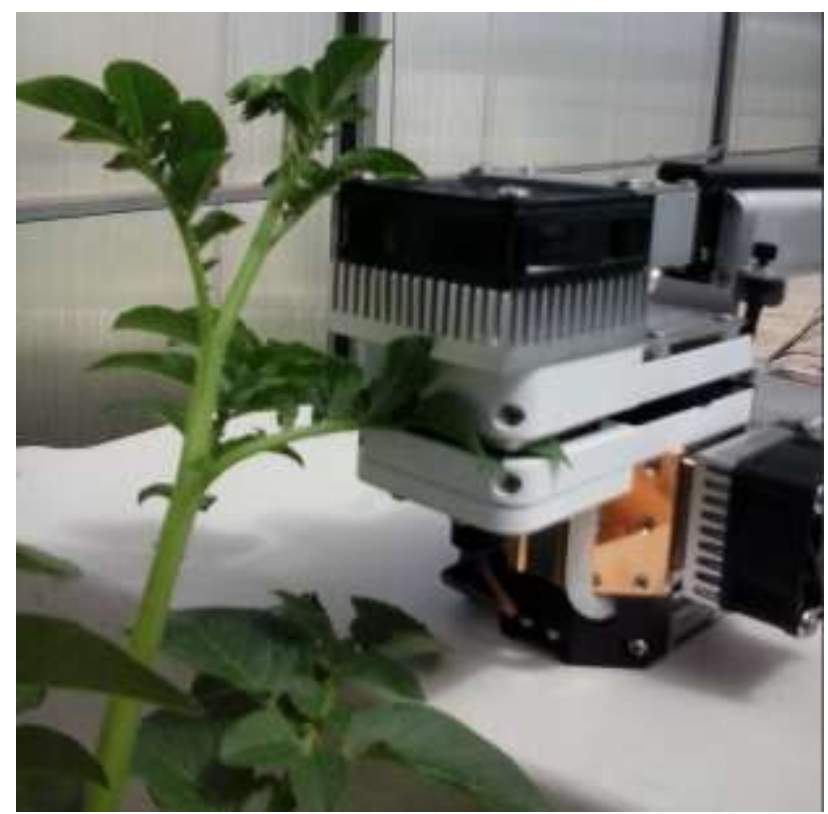

Fonte: Autores.

No mesmo dia, as plantas foram colhidas, lavadas em água corrente e divididas em parte aérea (folha e haste) e tubérculos. Para cada tratamento foi determinada a massa seca de tubérculos (MST) e total (MT) através da secagem em estufa com circulação forçada de ar a $60^{\circ} \mathrm{C}$ por 15 dias. Os dados foram submetidos à análise de variância (ANOVA) e as médias dos tratamentos comparadas pelo teste de Tukey $(\mathrm{p} \leq 0,05)$, utilizando-se o programa estatístico Sisvar® (Ferreira, 2000).

\section{Resultados e Discussão}

A variável produção de massa seca de tubérculos (MST) apresentou resposta significativa para o fator isolado de doses de $\mathrm{P}$ e clones de batata (Figura 2A e 2B). O aumento das doses de P proporcionou aumento da MST nas doses de 140 e $280 \mathrm{~kg}$ $\mathrm{P}_{2} \mathrm{O}_{5}$ ha $^{-1}$ (Figura 2A), sendo que, o clone Atlantic apresentou maior média em comparação aos demais (Figura 2B). Esse aumento na MST ocorreu devido o P estimular a tuberização, acelerar a maturação e aumentar a incidência de tubérculos maiores (Mesquita et al., 2011). Soratto et al. (2015), verificaram em batata cultivada sob elevado nível de P que a produção de massa seca de tubérculos foi $60 \%$ superior comparado as plantas cultivadas com baixo nível de P. A massa seca total, de folhas, hastes, raízes e tubérculos também foram maiores com o aumento dos níveis de P para cultivar de batata Kennebec (Fleisher et al., 2012). 
Figura 2. Efeitos de doses de fósforo sobre a matéria seca de tubérculos e total em clones de batata. Letras maiúsculas comparam a dose entre os genótipos. Letras minúsculas comparam cada genótipo entre as doses.
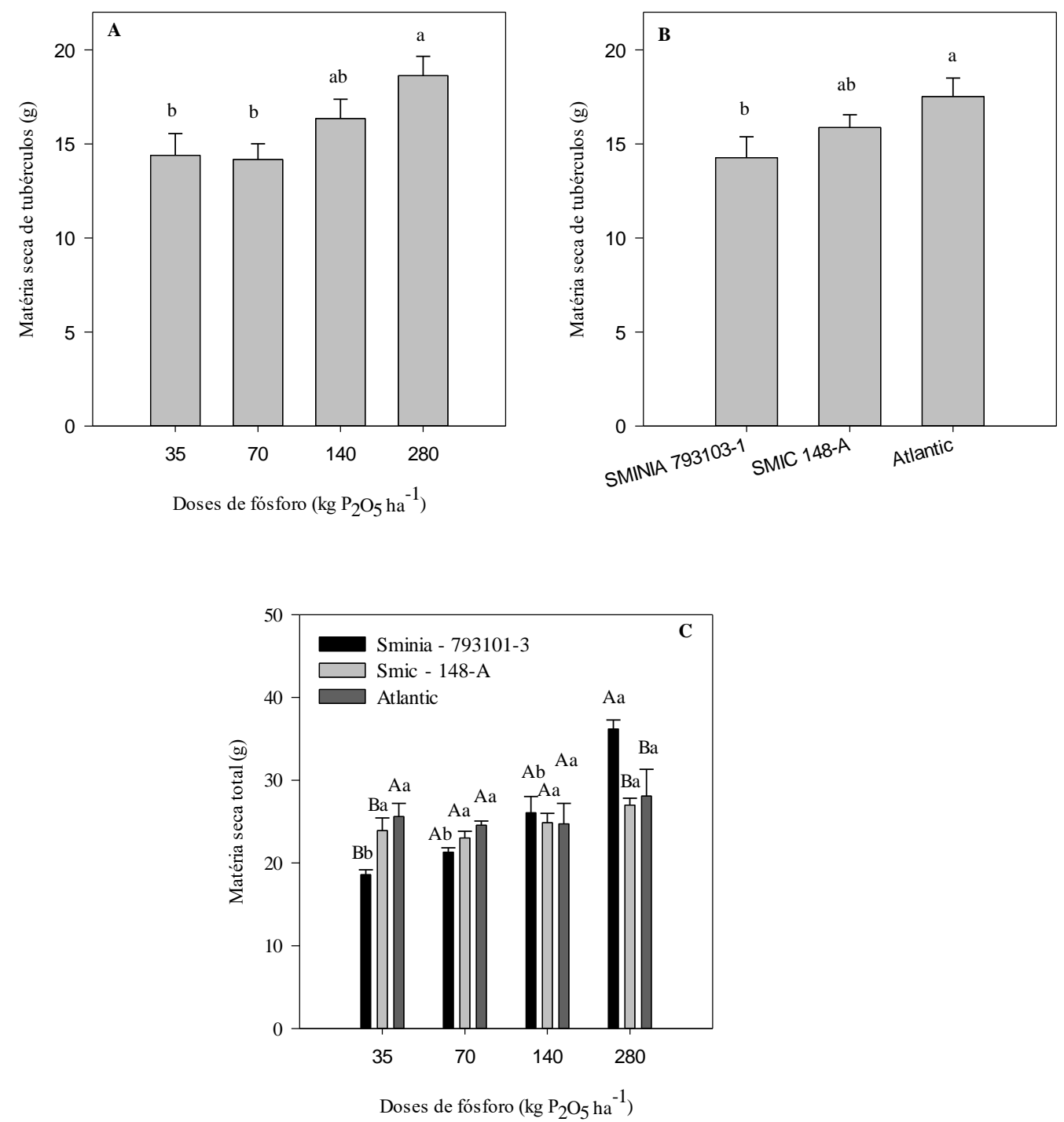

*Médias seguidas por letras diferentes diferem significativamente pelo teste Tukey $(\mathrm{p} \leq 0,05)$. Letra maiúscula compara os clones entre si em um mesmo tratamento. Letra minúscula compara cada clone nas diferentes doses de $\mathrm{P}\left(35,70,140 \mathrm{e} 280 \mathrm{mg} \mathrm{L}^{-1}\right)$. Fonte: Autores.

O P além de interferir na massa seca de tubérculos, pode influenciar também em várias outras características ligadas à qualidade destes, como no tamanho, peso específico e quantidade de amido dos tubérculos (Rosen et al., 2014), sendo dessa forma, um importante nutriente no manejo da cultura para obtenção de um ótimo retorno econômico. Observou-se também que o clone SMINIA 793101-3, apresentou, independente das doses de P estudadas menor MST em relação aos demais (Figura 2B), demonstrando menor alocação de fotoassimilados para a produção de tubérculos. Na batata, a distribuição da biomassa na planta ocorre principalmente entre a parte aérea e os tubérculos, atingindo de $68 \%$ a $84 \%$ da biomassa total produzida pela planta (Sharifi et al., 2005; Nunes et al., 2006).

Para a massa seca total (MT), houve efeito significativo na interação entre os fatores clones e doses de P estudadas (Figura 2C). Ocorreu variabilidade na eficiência e uso de P neste estudo, sendo o clone Atlantic que apresentou melhor resposta, pois mesmo na menor dose de $\mathrm{P}, 35 \mathrm{~kg} \mathrm{P}_{2} \mathrm{O}_{5}$ ha $^{-1}$, obteve maior MT quando comparado aos demais clones (Figura 2C), bem como apresentou maior MST (Figura 2B). De acordo com Thornton et al., (2019), a batata exibe uma variação 
genética considerável na eficiência de utilização de $\mathrm{P}$ (produção de tubérculos/unidade de $\mathrm{P}$ disponível). Curiosamente, verificou-se que o clone SMINIA 793101-3 na maior dose de $\mathrm{P}\left(280 \mathrm{~kg} \mathrm{P}_{2} \mathrm{O}_{5} \mathrm{ha}^{-1}\right)$ taxa de assimilação líquida de $\mathrm{CO} 2(A) \mathrm{e}$ condutância estomática (Gs) (Figura 3A e 3B), o que refletiu em maior MT (Figura 2B).

Figura 3. Efeitos de doses de fósforo sobre a taxa de assimilação líquida de $\mathrm{CO}_{2}(A)$, condutância estomática (Gs), eficiência do uso da água (EUA) e eficiência da carboxilação $(\mathrm{A} / \mathrm{C} i)$ em clones de batata. Letras maiúsculas comparam a dose entre os genótipos. Letras minúsculas comparam cada genótipo entre as doses.

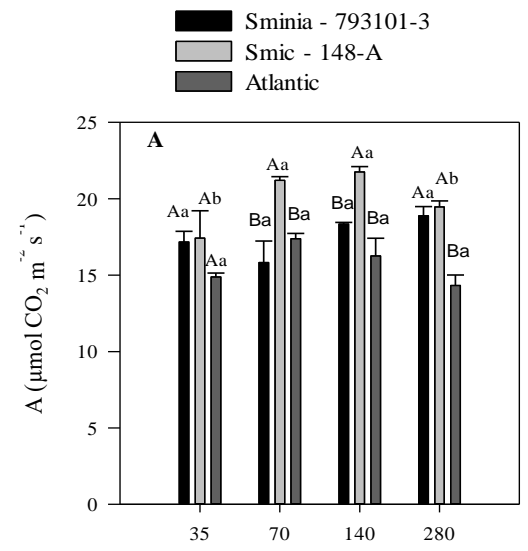

Doses de fósforo $\left(\mathrm{kg} \mathrm{P}_{2} \mathrm{O}_{5} \mathrm{ha}^{-1}\right)$

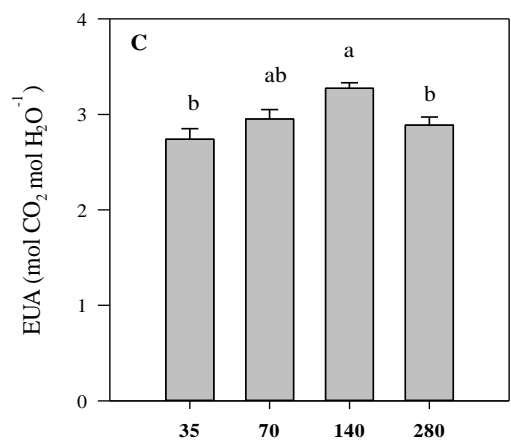

Doses de fósforo $\left(\mathrm{kg} \mathrm{P}_{2} \mathrm{O}_{5} \mathrm{ha}^{-1}\right)$

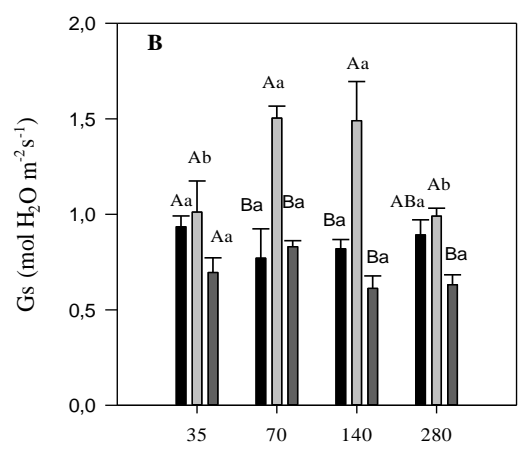

Doses de fósforo $\left(\mathrm{kg} \mathrm{P}_{2} \mathrm{O}_{5} \mathrm{ha}^{-1}\right)$

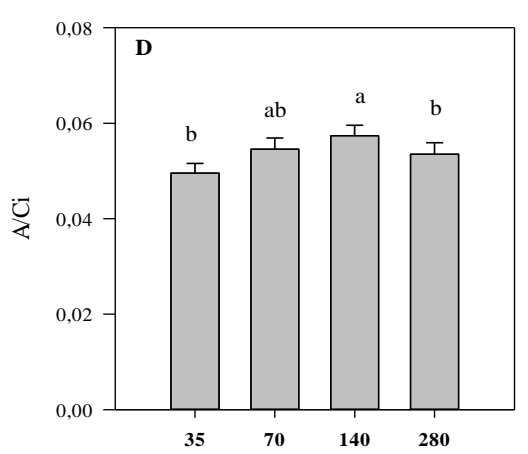

Doses de fósforo $\left(\mathrm{kg} \mathrm{P}_{2} \mathrm{O}_{5}\right.$ ha $\left.^{-1}\right)$

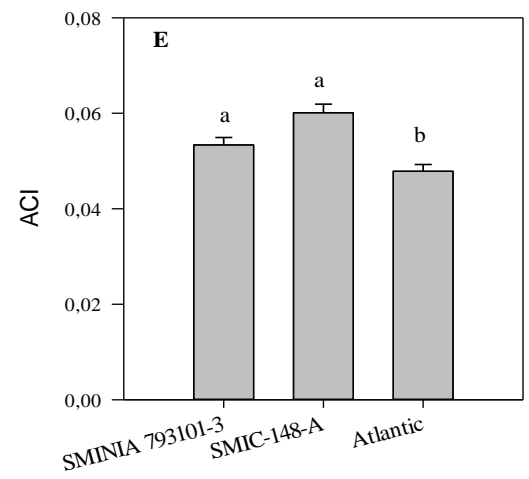

*Médias seguidas por letras diferentes diferem significativamente pelo teste Tukey $(\mathrm{p} \leq 0,05)$. Letra maiúscula compara os clones entre si em um mesmo tratamento. Letra minúscula compara cada clone nas diferentes doses de P $\left(35,70,140\right.$ e $\left.280 \mathrm{mg} \mathrm{L}^{-1}\right)$. Fonte: Autores. 
Desalegn et al. (2016), relataram que o aumento da disponibilidade de $\mathrm{P}$ aumentou o rendimento comercial e total de tubérculos da batata. Com adequado suprimento de $\mathrm{P}$, as plantas exibem incremento na assimilação de $\mathrm{CO}_{2}$, na eficiência de carboxilação e no processo fotoquímico, o que influencia no ganho de biomassa (Domingues et al., 2010; Warren, 2011). Estudos realizados por Sausen et al. (2020) demonstraram que o clone SMINIA 793101-3 não é eficiente em absorver P em baixa concentração, porém quando o nutriente é abundante ele responde eficientemente.

As variáveis $A$ e $G s$, apresentaram respostas à interação entre os fatores clones e doses de fósforo (P) estudadas (Figura 3A, 3B). Para eficiência de uso da água (EUA) houve resposta significativa apenas para o fator isolado de doses estudadas (Figura 3C). Já a eficiência instantânea de carboxilação da enzima ribulose-1,5-bifosfato carboxilase/oxigenasse $(\mathrm{A} / \mathrm{C} i)$ apresentou resposta significativa para o fator isolado clones e doses de $\mathrm{P}$ (Figura 3D e 3E).

Em relação a $A$ e $G s$, os clones quando expostos a baixa dose de $\mathrm{P}\left(35 \mathrm{~kg} \mathrm{P}_{2} \mathrm{O}_{5} \mathrm{ha}^{-1}\right)$ não apresentaram diferenças entre si. Entretanto, nas doses de 70, 140 e $280 \mathrm{~kg} \mathrm{P}_{2} \mathrm{O}_{5}$ ha $^{-1}$ o clone SMIC 148-A apresentou maior $A$ e $G s$ se comparado aos demais, sem diferir do clone SMINIA 793101-3 na maior dose de P $280 \mathrm{~kg} \mathrm{P}_{2} \mathrm{O}_{5}$ ha $^{-1}$ (Figura 3A e 3B). Analisando cada clone nas diferentes doses de P pode-se observar que o SMINIA 793101-3 e o Atlantic não diferiram para a A e Gs com o aumento da dose de P, enquanto, o SMIC 148-A apresentou maior $A$ e $G s$ nas doses 70 e $140 \mathrm{~kg} \mathrm{P}_{2} \mathrm{O}_{5}$ ha $^{-1}$ (Figura 3A e 3B). Assim como, encontrados em tomate que pertence à mesma família da batata (solanácea), Typha domingensis e Lotus japonicus em que a fotossíntese líquida aumentou quando estas foram expostas a níveis mais elevados de P (Groot et al., 2003; Santos et al., 2015; Thuynsma et al., 2016). Como também foi observado na batata cultivar Kennebec que apresentou redução na fotossíntese nos tratamentos com baixo P (Fleisher et al., 2012).

No aparelho fotossintético, o P desempenha papel de regulador na biossíntese de amido/sacarose e na ativação da Rubisco (ribulose 1,5 bisfosfato), uma vez que é usado para fosforilar intermediários do ciclo de Calvin e na disponibilidade de energia (Hernández \& Munné-Bosch, 2015). As diferenças relativas na $A$ entre os clones deste estudo podem ter ocorrido devido a variabilidade dos clones de batata quanto na eficiência de absorção e uso do P. E ainda, pelas diferenças entre os clones na alocação dos fotoassimilados, já que os açúcares produzidos pela fotossíntese são convertidos em amido e armazenados nos tubérculos.

Quanto a $G s$, esta indica o fechamento estomático e a regulação deste parâmetro é vital para a prevenção da perda de água e absorção do $\mathrm{CO}_{2}$ (Paiva et al., 2005). Pode-se observar neste estudo que as doses crescentes de $\mathrm{P}$ adicionadas, promoveram uma variação na $G s$ sendo o clone SMIC-148-A o que demonstrou ser responsivo ao $\mathrm{P}$, onde na dose $140 \mathrm{~kg}_{2} \mathrm{P}_{5}$ $\mathrm{ha}^{-1}$ apresentou maior $G s$, que consequentemente refletiu na $A$ (Figura $3 \mathrm{~A}$ e $3 \mathrm{~B}$ ). A deficiência de $\mathrm{P}$ pode resultar em diminuição do número e tamanho dos estômatos nas folhas contribuindo para redução da Gs e da A (Sarker et al., 2010).

Paralelo a isso verifica-se que a máxima EUA foi atingida na dose 70 e $140 \mathrm{~kg} \mathrm{P}_{2} \mathrm{O}_{5}$ ha $^{-1}$ (Figura 3C e 3D). A EUA está relacionada com a água perdida pela transpiração ao abrir os estômatos para capturar $\mathrm{CO}_{2}$, quanto menor a perda de água na captação de $\mathrm{CO}_{2}$, mais eficiente é o uso da água. Nossos resultados corroboram com Yi et al. (2019), que verificaram que plantas de batata apresentaram acréscimos na EUA quando expostas a aumentos na disponibilidade de $\mathrm{P}$ independente das condições de $\mathrm{CO}_{2}$ testadas.

Observou-se efeito direto do aumento nas doses de $\mathrm{P}$ (70 e $140 \mathrm{~kg} \mathrm{P}_{2} \mathrm{O}_{5} \mathrm{ha}^{-1}$ ) na funcionalidade da $\mathrm{A} / \mathrm{Ci}$ (Figura 3D), assim como em outros trabalhos que demonstraram que a disponibilidade do $\mathrm{P}$ afeta diretamente a ativação de algumas enzimas do ciclo de Calvin-Benson, como a A/Ci (Shubhra et al., 2004), refletindo, em um adequado desempenho da fase bioquímica (Iglesias et al., 2002). No entanto, independente das doses de P estudadas os clones SMINIA 793101-3 e SMIC148-A apresentaram a maior A/Ci (Figura 3E).

Como pode-se observar neste estudo o $\mathrm{P}$ é um nutriente que não limita somente a fotossíntese, mas também pode exercer efeito sobre o crescimento das plantas. O P é o segundo nutriente mineral mais limitante para a produção agrícola de 
batata após o nitrogênio, por isso, a utilização da dose adequada deste nutriente e o conhecimento de como diferentes clones de batata se comportam quanto a quantidade do nutriente disponibilizada, é de grande importância para garantir uma boa produção de tubérculos da cultura.

\section{Considerações Finais}

$\mathrm{O}$ aumento das doses de $\mathrm{P}$ proporcionou aumento da matéria seca dos tubérculos e total das plantas. Nesse estudo observou-se que os clones respondem de forma diferente ao incremento do P, o clone SMIC 148-A apresentou maior taxa de assimilação líquida do CO2 e condutância estomática nas doses de 70, 140 e 280 kg P2O5 ha-1. O SMINIA 793101-3 apresentou maior massa seca total, maior taxa de assimilação liquida $\mathrm{CO} 2$ e condutância estomática quando foi fornecida pela maior dose de P. No entanto, o clone Atlantic foi o que apresentou melhor eficiência do uso do P, pois mesmo com baixas doses desse nutriente obteve maior matéria seca de tubérculos e total. Esses resultados podem ser considerados para a seleção de clones de batata tolerantes à deficiência de $\mathrm{P}$.

\section{Agradecimentos}

Os autores agradecem ao CNPq pelo apoio financeiro e ao Programa de Melhoramento Genético da Batata da UFSM pela concessão dos genótipos.

\section{Referências}

Balemi, T. \& Schenk, M. K. (2009). Genotypic variation of potato for phosphorus efficiency and quantification of phosphorus uptake with respect to root characteristics. Journal of Plant Nutrition and Soil Science, 172, 669-677. 10.1002/jpln.200800246

Braun, H. Rezende Fontes, P. C. R., Busato, C. \& Cecon, P. R. (2011). Teor e exportação de macro e micronutrientes nos tubérculos de cultivares de batata em função do nitrogênio. Bragantia, 70, 50-57. 10.1590/S0006-87052011000100009

Comissão de química e fertilidade do solo - RS/SC. (2004). Manual de adubação e calagem para os Estados do Rio Grande do Sul e Santa Catarina. 10. Ed. Porto Alegre.

Embrapa. (2018). Sistema brasileiro de classificação de solos. 5. ed. - Rio de Janeiro: EMBRAPA-SPI, 356p. https://www.embrapa.br/solos/sibcs

Dechassa, N., Schenk, M. K., Claassen, N. \& Steingrobe, B. (2003). Phosphorus efficiency of cabbage (Brassica oleraceae L. var. capitata), carrot (Daucus carota L.), and potato (Solanum tuberosum L.). Plant Soil, 250,215-224. 10.1023/A:1022804112388

Desalegn, R., Wakene, T., Dawit, M. \& Tolessa, T. (2016). Effects of Nitrogen and Phosphorus Fertilizer Levels on Yield and Yield Components of Irish Potato (Solanum tuberosum) at Bule Hora District, Eastern Guji Zone, Southern Ethiopia. International Journal of Agricultural Economics, 1 , $71-77$.

Domingues, T. F., Meir, P., Feldpausch, T. R., Saiz, G., Veenendaal, E. M., Schrodt, F., Bird, M., Djagbletey, G., Hien, F., Compaore, H., Diallo, H., Grace, J. \& Lloyd, J. (2010). Co-limitation of photosynthetic capacity by nitrogen and phosphorus in West capacity by nitrogen and phosphorus in West Africa Woodlands. Plant, Cell and Environment, 33,959-980. 10.1111/j.1365-3040.2010.02119.x

Fleisher, D. H. Wang, Q., Timlin, D. J., Chun, J. \& Reddy, V. R. (2012). Response of Potato Gas Exchange and Productivity to Phosphorus Deficiency and Carbon Dioxide Enrichment. Crop Science, 52, 1803-1815. 10.2135/cropsci2011.09.0526

Ferreira, D.F. (2000). Análises estatísticas por meio do Sisvar para Windows versão 4.0. In: Reunião anual da região brasileira da sociedade internacional de biometria, SIB, 255-258.

Groot, C. C., Boogaard, R. V. D., Marcelis, L. F. M., Harbinson, J. \& Lambers, H. (2003). Contrasting effects of N and P deprivation on the regulation of photosynthesis in tomato plants in relation to feedback limitation. Journal of Experimental Botany, 54, 1957-1967. 10.1093/jxb/erg193

Haan, S. \& Rodriguez, F. (2016). Potato Origin and Production. In: Singh, J. \& Kaur, L. (ed.) Advances in Potato Chemistry and Technology. 10.1016/B978$0-12-800002-1.00001-7$

Heldwein, A. B., Streck, N. A. \& Bisognin, D. A. (2009). Batata. In: Monteiro, J. E. B. A. (ed.) Agrometeorologia dos cultivos: o fator meteorológico na produção agrícola. Brasília: Instituto Nacional de Meteorologia, .91-109.

Hernández, I. \& Munné-Bosch, S. (2015). Linking phosphorus availability with photo-oxidative stress in plants. Journal of Experimental Botany, 66, 28892900. $10.1093 / \mathrm{j} x \mathrm{~b} / \mathrm{erv} 056$

Iglesias, D. J., Lliso, I., Tadeo, F. R. \& Talon, M. (2002). Regulation of photosynthesis through source: sink imbalance in citrus is mediated by carbohydrate content in leaves. Physiologia Plantarum, 116, 563-572. 10.1034/j.1399-3054.2002.1160416.x 
Jacob, J. \& Lawlor, D. W. (1992). Dependence of photosynthesis of sunflower and maize on phosphate supply, ribulose-1,5-bisphosphate carboxylase/oxygenase activity, and ribulose-1,5-bisphosphate pool size. Plant Physiology, 98,801-807. http://dx.doi.org/10.1104/pp.98.3.801

Koch, M., Naumann, M., Pawelzik, E., Gransee, A. \& Thiel, H. (2019). The importance of nutrient management for potato production Part I: Plant nutrition and yield. Potato Research. 10.1007/s11540-019-09431-2

Mesquita, H. A., Paula, M. B., Venturin, R. P., Pádua, J. G. \& Yuri, J. E. (2011). Fertilização da cultura da batata. In: Zambolim, L. (ed.). Produção integrada da batata. Viçosa: Universidade Federal de Viçosa, Departamento de Fitopatologia, 1,351-380.

Nunes, J. C. S., Fontes, P. C. R., Araújo, E. F. \& Sediyama, C. (2006). Potato plant growth and macronutrients uptake as affected by soil tillage and irrigation systems. Pesquisa Agropecuária Brasileira, 41,1787-1792. 10.1590/S0100-204X2006001200014

Paiva, A. S., Fernandes, E. J., Rodrigues, T. J. D. \& Turco, J. E. P. (2005). Condutância estomática em folhas de feijoeiro submetido a diferentes regimes de irrigação. Engenharia Agrícola, 25,161-169. 10.1590/S0100-69162005000100018

Pieters, A. J., Paul, M. J. \& Lawlor, D. W. (2001). Low sink demand limits photosynthesis under Pi deficiency. Journal of Experimental Botany, 52,1083-109. $10.1093 /$ jexbot/52.358.1083

Plenet, D., Mollier, A. \& Pellerin, S. (2000). Growth analysis of maize field crops under phosphorus deficiency. II. Radiation-use efficiency, biomass accumulation and yield components. Plant and Soil, 224,259-272. 10.1023/A:1004835621371

Rosen, C. J., Kelling, K. A., Stark, J. C. \& Porter, G. A. (2014). Optimizing phosphorus fertilizer management in potato production. American Journal of Potato Research, 91,145-160. 10.1007/s12230-0149371-2.10

Santos, K. R., Pereira, M. P., Ferreira, A. C. G., Rodrigues, A. C. A., Castro, E. M., Corrêa, F. F. \& Pereira, F. J. (2015). Typha domingensis Pers. growth responses to leaf anatomy and photosynthesis as influenced by phosphorus. Aquatic Botany, 122, 47-53. 10.1016/j.aquabot.2015.01.007

Santos, M. G., Ribeiro, R. V., Oliveira, R. F., Machado, E. C. \& Pimentel, C. (2006). The role of inorganic phosphate on photosynthesis recovery of common bean after a mild water deficit. Plant Science, 170,659-664. 10.1016/j.plantsci.2005.10.020

Sarker, B. C., Karmoker, J. L. \& Rashid, P. (2010). Effects of phosphorus deficiency on anatomical structures in maize (Zea mays L.). Bangladesh Journal of Botany, 39(1), 57-60. 0.3329/bjb.v39i1.5527

Sausen, D., Mambrin, R. B., Cassanego, D. B., Alves, J. S., Pereira, A. S., Dorneles, A. O. S., Bernardy, K. \& Schwalbert, R. (2020). Produção de batata a partir de micropropagação e de minitubérculos sob níveis de fósforo em solução nutritiva. Brazilian. Journal of Development, 6(2), 6648-6657. 10.34117/bjdv6n2-097

Sharifi, M., Zebarth, B. J., Hajabbasi, M. A. \& Kalbasi, M. (2005). Dry matter and nitrogen accumulation and root morphological characteristics of two clonal selections of 'Russet Norkotah' potato as affected by nitrogen fertilization. Journal of Plant Nutrition, 28, 2243-2253. 10.1080/01904160500323552

Shen, J., Yuan, L., Zhang, J., Li, H., Bai, Z., Chen, X., Zhang, W. \& Zhang, F. (2004). Phosphorus dynamics: From soil to plant. Plant Physiology, 156,9971005. 10. 1104/ pp. 111. 175232

Shubhra, B., Dayal, J., Goswami, C. L. \& Munjal R. et. al. D. (2004). Influence of phosphorus application on water relations, biochemical parameters and gum content in cluster bean under water deficit. Biologia Plantarum, 48,445-448. 10.1023/B:BIOP.0000041101.87065.c9

Soratto, R. P., Pilon, X. C., Fernandes, A. M. \& Moreno, L. A. (2015). Phosphorus Uptake, Use Efficiency, and Response of Potato Cultivars to Phosphorus Levels. Potato Research, 58,121-134. 10.1007/s11540-015-9290-8

Thuynsma, R., Kleinert, A., Kossmann, J., Valentine, A. J. \& Hills, P. N. (2016). The effects of limiting phosphate on photosynthesis and growth of Lotus japonicus. South African Journal of Botany, 104,244-248. 10.1016/j.sajb.2016.03.001

Thornton, M. K., Novy, R. G. \& Stark, J. C. (2014). Improving phosphorus use efficiency in the future. American Journal of Potato Research, 91,175-179. Obtido de https://doi.org/10.1007/s12230-014-9369-9

Wang, X., Shen, J. \& Liao, H. (2010). Acquisition or utilization, which is more critical for enhancing phosphorus efficiency in modern crops? Plant Science, 179,302-306. 10.1016/j.plantsci.2010.06.007

Warren, C. R. (2011). How does P affect photosynthesis and metabolite profles of Eucalyptus globulus? Tree Physiology, 31,727-739. 10.1093/tre e phys/tpr 064

Yi, Y., Sugiura D., \& Yano, K. (2019). Quantifying Phosphorus and Water Demand to Attain Maximum Growth of Solanum tuberosum in a $\mathrm{CO}_{2}-$ Enriched Environment. Frontiers in Plant Science, 10, 1417. 10.3389/fpls.2019.01417 